\title{
EDIFİCATE
}

I Congreso de Escuelas de Edificación y Arquitectura Técnica de España

València, 4 y 5 de noviembre de 2021

Escuela Técnica Superior de Ingeniería de Edificación

Universitat Politècnica de València

Doi: https://doi.org/10.4995/EDIFICATE2021.2021.13553

\section{Innovación pedagógica y aprendizajes en las asignaturas de Construcción en el Grado en Arquitectura Técnica de la Universidad de Burgos}

\section{Pedagogical innovation and learning in Construction subjets in the Degree in Technical Architecture of the University of Burgos}

\author{
Carmelo Muñoz Ruiperez ${ }^{a}$, Marina Muñoz-Arranz ${ }^{\mathrm{b}}$; Javier Garabito López ${ }^{\mathrm{c}}$; Francisco \\ Fiol Olivand \\ Universidad de Burgos \\ a cmruip@ubu.es, b munozarranz@gmail.com , cjgarabito@ubu.es, ${ }^{d}$ ffiol@ubu.es
}

\begin{abstract}
One of the main challenges of higher education is knowing how to adapt the training of students to the continuous need for innovation and research, a fundamental pillar in our education, being used as a differentiating and valuable element. The arrival of the Bologna plan provided new changes in university management and planning, achieving classes with a higher practical percentage, thus complementing the scientific or regulatory and technical aspects. In Construction subjects, the cooperative teaching-learning method is used, providing the student with a greater degree of autonomy, involvement and responsibility. Working cooperatively with students in construction subjects is mainly focused on practical classes where study cases are presented in which a simulation of real problems is carried out before which the student must propose and select solutions among which consider more appropriate
\end{abstract}

Keywords: Innovation, research, teaching methodology, teaching-learning.

\section{Resumen}

Uno de los principales desafíos de la educación superior es saber adaptar la formación del alumnado a la continua necesidad de innovación e investigación, un pilar fundamental en nuestra educación, empleándose como elemento diferenciador y de valor. La llegada del plan Bolonia proporcionó nuevos cambios en la gestión y planificación universitaria, logrando clases con mayor porcentaje práctico, consiguiendo de esta manera complementar el aspecto científico o normativo y el técnico. En las asignaturas de Construcción se emplea el método de enseñanza-aprendizaje cooperativo, proporcionando al 
alumnado un mayor grado de autonomía, implicación y responsabilidad. El trabajo con los estudiantes de manera cooperativa en las asignaturas de construcción se materializa sobre todo en las clases prácticas donde se plantean casos de estudio en los que se realiza una simulación de problemas reales ante los que el estudiante debe plantear y seleccionar soluciones entre las que considere más apropiada.

Palabras clave: Innovación, investigación, metodología docente, enseñanzaaprendizaje. 


\section{Introducción}

\subsection{El concepto de innovación en la Universidad}

Uno de los principales desafíos de la educación superior es saber adaptar la formación del alumnado a la continua necesidad de innovación e investigación (Rué, 2007), un pilar fundamental en nuestra educación, empleándose como elemento diferenciador y de valor.

Es fundamental describir, analizar y concretar el concepto de innovación educativa, dado que este ha ido evolucionando a medida que lo hacia la sociedad, adaptándose a las necesidades y a los constantes cambios de esta.

El concepto de innovación surge en el ámbito industrial, y poco a poco, se ha ido adaptando a la sociedad de la información (Margalef y Arenas, 2006), llegando a convertirse en un elemento propio dentro de las universidades a nivel mundial (Gairín, 2001; Quizhpe, Gómez y Aguilar, 2016).

Actualmente se habla de innovación más que nunca, pero, estas innovaciones deben consolidarse, evaluarse y adaptarse a las nuevas necesidades, siendo necesario un cambio de mentalidad por parte de todos los agentes principales en la educación, profesorado y alumnado (López-Martín, Días y Tiana, 2017).

El cambio de actitudes debe ir acompañado de un cambio en la forma de pensar y actuar, y que se fundamenta en nuevos objetivos, competencias, metodologías y estrategias de evaluación.

El alumnado debe convertirse en el principal agente en los procesos de enseñanzaaprendizaje, donde el docente actua de guía de los conocimientos y anima a los estudiantes a involucrarse en la educación, alentándoles a transformar el aprendizaje, cambiándolo, acomodándolo, mejorándolo, modificándose uno en el mismo proceso, convirtiéndose en líderes del cambio (García-Retamero, 2010; Salinas, 2004).

Nosotros como docentes, debemos valorar la innovación, la exploración, la creatividad... convirtiendo el proceso de enseñanza-aprendizaje en la comprensión, experimentación, resolución de problemas e indagación.

Como decía Piaget, debemos dejar que los estudiantes aprendan, que sean ellos quienes descubran, porque de esta manera, lograremos un mayor aprendizaje, una mayor autonomía, un mayor desarrollo de habilidades y un mayor crecimiento personal.

Podemos definir como innovación al proceso orientado a la mejora de un curso, un departamento o la persona que conlleva una planificación, una organización y un esfuerzo en busca del cambio (Gros y Lara, 2009), convirtiéndose en la llave para lograr una cohesión 
dentro de la educación, la formación y el crecimiento como docentes, y como consecuencia del alumnado.

\subsection{Innovación en las asignaturas de Construcción del Grado de Arquitectura Técnica en la Universidad de Burgos}

Desde la implantación en el curso 2010-2011 del Grado de Arquitectura Técnica, en las asignaturas de Construcción, hemos buscado la mejora y el aprendizaje, adaptando las guías docentes y los programas de las asignaturas a los cambios que surgían en la normativa, así como a las necesidades de los estudiantes.

Todo ello, teniendo en cuenta la relación entre sociedad-empresa-universidad, pilar fundamental para lograr un proceso enseñanza-aprendizaje y una formación más práctica integral y funcional.

La llegada del plan Bolonia proporcionó nuevos cambios en la gestión y planificación universitaria, logrando clases con mayor porcentaje práctico, consiguiendo de esta manera complementar el aspecto científico o normativo y el técnico.

Tabla 1: Asignaturas de Construcción en el Grado de Arquitectura Técnica en UBU

\begin{tabular}{|c|c|c|c|c|}
\hline ASIGNATURAS & CURSO & SEMESTRE & CRÉDITOS & CONTENIDOS \\
\hline Construcción I & $1^{a}$ & 1 & 6 & $\begin{array}{l}\text { Iniciación al conocimiento básico de los procesos constructivos. } \\
\text { Terreno. Técnicas y sistemas constructivos en cimentacione. } \\
\text { Sistemas de contención. }\end{array}$ \\
\hline Construcción II & $1^{\circ}$ & 2 & 6 & $\begin{array}{l}\text { Evolución histórica de las técnicas de construcción. Obras de } \\
\text { fábrica (resistentes y particiones). Introducción a las cubiertas }\end{array}$ \\
\hline Construcción III & $2^{\circ}$ & 1 & 6 & $\begin{array}{l}\text { Elementos constructivos realizados con hormigón. Cimentaciones. } \\
\text { Muros. Estructuras a base de muros. Entramados. Escaleras. } \\
\text { Prefabricación. EHE08. }\end{array}$ \\
\hline Construcción IV & $2^{a}$ & 2 & 6 & Canteria. Carpinteria de armar. Estructuras de acero y mixtas. EAE \\
\hline Construcción V & $3^{a}$ & 1 & 6 & Cubiertas. Fachadas. Revestimientos. Acabados \\
\hline
\end{tabular}

La implicación e intervención del alumnado en las sesiones también tomo un papel mucho más importante, logrando que fueran ellos quienes a través de continuas retroalimentaciones fueran indicando las necesidades de mejora y repaso en ciertos contenidos.

Es por ello, que desde las asignaturas de Construcción del Grado de Arquitectura Técnica nos planteamos seguir la siguiente estructura en la realización, programación y desarrollo del curso académico.

Como metodología docente, las asignaturas de Construcción cuentan con horas teóricas y prácticas, dividiéndose estas en prácticas de pizarra y prácticas en el taller de construcción, 
de forma que se complementa el aspecto científico o normativo y el técnico, posibilitando al estudiante una mejor comprensión de lo explicado, ya que en construcción la realidad teórica y práctica van indisolublemente unidas.

Para el desarrollo de las asignaturas de Construcción del grado Arquitectura Técnica se han elaborado unos materiales que se publican en la plataforma Moodle y que están a disposición de los estudiantes. Estos contenidos se van actualizado en virtud de las cambios normativos o nuevas técnicas constructivas que aparecen en el mercado. La normativa vigente y videos de construcción forman parte de su aprendizaje, y están a disposición de los estudiantes a través de la plataforma. Se pretende con todos estos materiales fomentar la actitud investigadora y que los estudiantes usen de forma correcta las tecnologías de la información que se disponen.

En las asignaturas de Construcción durante las presentaciones que se realizan el primer día de clase se les proporciona a los estudiantes un programa completo con toda la información necesaria para el desarrollo de estas, se les plantean los objetivos generales, la planificación con las clases teóricas, clases prácticas y de taller, la bibliografía seleccionada, el calendario de entrega de las prácticas y los controles que se efectuarán. Es importante que el estudiante conozca de antemano todos los aspectos de la asignatura.

La función de las tutorías es la de consulta y orientación académica, clarificando las dudas sobre la materia impartida y permitiendo afrontar la enseñanza de forma individualizada según las necesidades personales de cada estudiante, este recurso didáctico permite el intercambio de ideas entre el estudiante y el profesor, ofreciéndole retroalimentación sobre su trabajo. Las tutorías se realizan tanto de forma presencial como online.

La formación de las asignaturas de Construcción se complementa con la realización de conferencias, estas son impartidas por empresas del sector de la construcción donde se pretende que el estudiante conozca la realidad de la vida profesional, y fomentar y motivar el interés del estudiante por determinadas materias.

La realización de controles cada 3-4 semanas y que el contenido de estos sea propugnado por los estudiantes intenta fomentar la motivación de los estudiantes promoviendo el protagonismo de estos y la obtención de la recompensa de no tener que presentarse al examen final en función de la calificación de los controles.

Otra de las bases del aprendizaje es la inmersión tecnológica, que aumenta con la adaptación de escenario educativo que hemos tenido que realizar con la llegada de la pandemia, lo que nos ha permitido integrar aún más las nuevas tecnologías en el aula, convirtiendo las TICs en nuestro nuevo aliado en la educación.

Como dice Salinas (2004), la innovación docente va de la mano con las tecnologías de la información y la comunicación, las cuales, se encuentran integradas en los procesos de 
formación, dando acceso a nuevos usuarios, apoyando los cambios en los procesos de enseñanza-aprendizaje y haciendo estos aún más flexibles.

\section{Objetivos}

Los principales objetivos que se pretenden lograr introduciendo la innovación y las metodologías pedagógicas en las asignaturas de Construcción del Grado de Arquitectura Técnica en la Universidad de Burgos son los siguientes:

- Lograr una mayor autonómica, resolución de problemas, creatividad, formación en el alumnado.

- Proporcionar mayor importancia a la figura del estudiante en el desarrollo de las clases y las asignaturas.

- Reflexionar sobre el cambio de metodología pedagógica que estamos implantando en las asignaturas de Construcción.

- Cambiar la mentalidad de los agentes educativos en el proceso de enseñanzaaprendizaje

- Apoyar la importancia de la formación permanente del profesorado universitario.

- Aumentar la motivación docente por un aprendizaje teórico-práctico, activo y significativo

- Mejora de las tasas de éxito

\section{Desarrollo de la innovación}

En el desarrollo de los cursos, asignaturas y sesiones pretendemos que los estudiantes adquieran una serie de competencias, habilidades y contenidos, pero siempre bajo el objetivo de lograr futuros profesionales.

Se considera metodología pedagógica activa o innovadora a aquellas actividades o conjuntos de procesos que obligan al cambio de mentalidad tradicional, poniendo el enfoque en los procesos de enseñanza-aprendizaje y proporcionando una mayor importancia e implicación del alumnado en el desarrollo de estas (Silva y Maturana, 2017).

Las diferentes metodologías innovadoras se basan en el modelo centrado en el aprendizaje, que nos permiten lograr una mayor adquisición de los procesos de enseñanza-aprendizaje y un mayor grado de autonomía, implicación y responsabilidad del alumnado (Gallardo, Morara y García, 2015).

La propuesta de metodologías activas en las asignaturas de Construcción propicia un cambio de paradigma tradicional, incorporando enseñanzas participativas, planteando problemas en donde se involucran diferentes áreas de conocimiento de manera interdisciplinar, buscando un proceso dinámico de aprendizaje. 


\subsection{Aprendizaje cooperativo e interdisciplinar}

El trabajo en equipo es uno de los pilares en nuestra sociedad, y más en la educación, es por ello, que debemos aprender a trabajar en grupo, siendo aplicable tanto al alumnado como al profesorado.

La estrategia de aprendizaje cooperativo nos permite llevar a cabo actividades tanto dentro como fuera del aula convirtiéndolas en experiencias de crecimiento tanto social como de aprendizaje, en la que los diferentes integrantes buscan un objetivo común aportando lo mejor de ellos (García Ruiz y González, 2013).

El trabajo con los estudiantes de manera cooperativa en las asignaturas de construcción se centra sobre todo en las clases prácticas donde se plantean casos de estudio en los que se realiza una simulación de problemas reales ante los que el estudiante debe plantear y seleccionar soluciones entre las que considere más apropiadas.

Las prácticas deben ser presentadas y defendidas por grupos de trabajo (máximo 3 personas por grupo), debiendo los otros grupos formular cuestiones sobre aspectos del trabajo presentado, logrando una heteroevaluación entre los propios estudiantes, aprendiendo los unos de los otros. Se promueve el espíritu emprendedor del estudiante, el pensamiento crítico y su autonomía, ya que son ellos, quienes deben defender sus presentaciones e ideas.

En las prácticas los estudiantes comparten sus dudas y aplican lo aprendido como de lluvia de ideas, logrando una cohesión grupal y una mayor satisfacción en los resultados.

Esta técnica también les proporciona habilidades de trabajo en grupo, resolución de conflictos, interdependencia positiva, y valores como la responsabilidad, el respeto y la confianza.

El trabajo del profesorado es multidisciplinar, es decir, que involucra el trabajo de diferentes profesionales de diferentes disciplinas y departamentos. Logrando así una mayor amplitud de conocimientos y perspectivas a la hora de transmitir las clases y de desarrollar las guías docentes (López, Corrales, Corchuelo y Blanco, 2015).

\subsection{Educación práctica}

Con esta metodología se pretende acercar al estudiante la realidad de las empresas, de la vida profesional, haciendo que los estudiantes sean conscientes de las necesidades laborales de estas.

A nuestro juicio una de las funciones principales de un profesor es la de proporcionar-entrenar nuevas estrategias que ayuden en la adquisición de conocimientos, este aspecto se ve materializado en las prácticas donde se plantean casos de estudio en los que se realiza una 
simulación de problemas reales ante los que el estudiante debe plantear y seleccionar soluciones entre las que considere más apropiadas. Las prácticas se desarrollan mediante trabajo colaborativo, estas deben ser presentadas y defendidas por los grupos de trabajo (máximo 3 personas por grupo), debiendo los otros grupos formular cuestiones sobre aspectos del trabajo presentado, logrando una heteroevaluación entre los propios estudiantes, aprendiendo los unos de los otros. Se promueve el espíritu emprendedor del estudiante, el pensamiento crítico y su autonomía, ya que son ellos, quienes deben defender sus presentaciones e ideas.

Los profesores que impartimos las asignaturas de construcción contamos con una amplia experiencia profesional, proporcionando a los estudiantes una imagen realista de los problemas que se pueden plantear en un futuro, así como fomentar habilidades en cuanto a la resolución de problemas de la vida real.

\subsection{Aprendizaje activo}

La metodología activa pretende la integración activa de los estudiantes en la planificación y desarrollo de las sesión y en la adquisición de aprendizajes.

La filosofía parte del cambio de papel del profesor, quien actúa como guía y orienta en el desarrollo de los aprendizajes, aportando mayor importancia a los estudiantes, y en donde se fomenta su aprendizaje autónomo, la resolución de problemas y la adquisición de habilidades y competencias pedagógicas (Morera et al, 2013).

Este cambio también repercute en los estudiantes, quienes deben cambiar la mentalidad de receptores de conocimiento, convirtiéndose en investigadores e indagadores de información constante, es decir, en sujetos activos de aprendizaje.

En las asignaturas de Construcción del Grado de Arquitectura Técnica pretendemos que nuestro estudiantes sean los protagonistas del aprendizaje, es por ello, que en la primera clase se dedica a la explicación de lo que se va a impartir en cada asignatura, cómo se estructura esta, los objetivos, habilidades y competencias que se pretende que adquieran, cómo se va a impartir la docencia (teóricas, prácticas, taller), los profesores que van a impartirla, los criterios de evaluación, para que el estudiante tenga una idea global de la asignatura y puedan reflexionar en cuanto a su progreso y planificación.

Durante el desarrollo de todas sesiones, los estudiantes tienen un papel activo, siguiendo la filosofía "aprendiendo a hacer", donde tanto profesores y estudiantes nos encontramos en un continuo feedback de aprendizajes.

Dedicamos la última clase a departir con los estudiantes todos aquellos aspectos de la asignatura que les han parecido más o menos interesantes, en resumen, determinar el grado de satisfacción de estos con la asignatura, realizando una autoevaluación que permite posteriormente junto con las encuentas regladas que realiza la Universidad de Burgos 
mejorar y complementar o trabajar respecto a ello, lo que nos ha permitido introducir cambios en las asignaturas que han hecho que estas evolucionen.

Las tutorías con los estudiantes son fundamentales en el proceso enseñanza-aprendizaje, y muy enriquecedoras tanto para los estudiantes como para nosotros como profesores. La figura del docente debe ir más allá de ser un mero transmisor de conocimientos, sirviendo las tutorías como una labor de apoyo y asesoramiento para desarrollar una actividad complementaria a la didáctica del aula.

\section{Resultados}

Los cambios que se han ido realizando (prácticas en grupo, heteroevaluación de las mismas por los propios estudiantes, realización de controles formulados por ellos mismos, etc..) han sido del agrado de los estudiantes que han respondido con entusiasmo a los mismos en las reflexiones que se han realizado con ellos, siendo los resultados académicos muy elocuentes, con tasas de éxito muy satisfactorias.

Tabla 2: Tasas de éxito en los últimos 4 cursos en Construcción III y IV

\begin{tabular}{|l|c|c|c|c|}
\hline \multirow{2}{*}{ ASIGNATURAS } & \multicolumn{4}{|c|}{ TASA DE ÉXIIO } \\
\cline { 2 - 5 } & Curso 17-18 & Curso 18-19 & Curso 19-20 & Curso 20-21 \\
\hline Construcción III & $62,50 \%$ & $60 \%$ & $100 \%$ & $72,70 \%$ \\
\hline Construcción IV & $81 \%$ & $87,50 \%$ & $100 \%$ & $95 \%$ \\
\hline
\end{tabular}

Pero todavía existen factores que en nuestra opinión influyen negativamente en los resultados académicos de alguna asignatura, como en el caso de la asignatura de Construcción III, que se imparte el primer semestre en el segundo curso, y determinados contenidos fundamentales para el desarrollo de esta asignatura se imparten en el siguiente semestre, este aspecto ha sido sancionado por estudiantes repetidores, en las reflexiones que se mantienen con los estudiantes.

\section{Conclusiones y aprendizajes}

La llegada de la innovación a la educación superior trae consigo un necesario cambio de ideologías y paradigmas, así como la necesaria reformulación pedagógica dentro del Grado de Arquitectura Técnica.

En la formación de nuestros estudiantes es necesario el aprendizaje de fórmulas, técnicas, procedimientos... pero también debemos tener la perspectiva humanística, somos personas que tratamos con personas y formamos a personas. 
Lo que nos lleva un cambio necesario de las guías docentes, en los contenidos de las asignaturas, y en las guías docente que en muchas ocasiones se han estacado en la tradicionalidad.

Nosotros como docentes debemos propiciar la innovación dentro de nuestros propios estudios, en nuestras aulas y en nuestros estudiantes. Para ello, debemos realizar un cambio en las metodologías, haciéndolas más innovadoras y pedagógicas.

La figura del docente debe estar en continua formación y crecimiento, adaptándose a los avances de la sociedad, generando una necesidad continua de evolución y crecimiento.

Debemos cambiar el enfoque de enseñanza por el enfoque de enseñanza-aprendizaje, centrándonos más en el aprendizaje de los estudiantes, dándoles mayor importancia, responsabilidad y autonomía, generando futuros profesionales cualificados con gran cantidad de recursos, cualidades y habilidades.

Estos nuevos cambios han supuesto grandes dificultades, haciéndonos volver a aprender, y proporcionándonos esa necesidad de seguir avanzando, aunque todavía nos queda mucho por aprender. El conocimiento de estas nuevas técnicas y metodologías por parte de los docentes nos permiten influir positivamente en la calidad educativa e incentivar en los estudiantes la construcción del conocimiento con reflexión, análisis y creatividad.

Las nuevas metodologías han proporcionado a las asignaturas de construcción donde se han aplicado un nuevo aire de adaptación, de crecimiento y de evolución. Permitiéndonos reflexionar sobre la necesidad de cambio en la práctica docente.

\section{Bibliografía}

GAIRÍN, J. (2001). "La innovación en la Universidad” en Revista de Investigación Educativa, vol 19, issue 2, p 635-640.

GALLARDO, B., MORERA, I., \& GARCÍA, E. (2015). "Metodología innovadora en la universidad. Sus efectos sobre los procesos de aprendizaje de los estudiantes universitarios" en Anales de Psicología, vol 31, issues 3, p 901-915.

GARCÍA-RETAMERO, J. (2010). "De profesor tradicional a profesor innovador" en Revista digital para profesionales de la enseñanza, issue 11.

GARCÍA-RUIZ, R.M. \& GONZÁLEZ, N. (2013). "El aprendizaje cooperativo en la universidad. Valoración de los estudiantes respecto a su potencialidad para desarrollar competencias" en Revista Iberoamérica para la Investigación y el Desarrollo Educativo, vol 4, issue 7, p 106-128.

GROS, B. \& LARA, P. (2009). "Estrategias de innovación en la educación superior: el caso de la Universitat Oberta de Catalunya" en Revista Iberoamericana de Educación, issues 49, p 223-245. 
LÓPEZ, M.J., CORRALES, N.M., CORCHUELO, B. \& BLANCO, M.A. (2015). "Interdisciplinariedad a través del aprendizaje cooperativo para adquisición de competencias" en Campo Abierto, vol 34, issue $1, \mathrm{p} 103-121$.

LÓPEZ-MARTíN, R., DIAS, P. \& TIANA, A. (2017).(Eds.), “ E-Innovación en la educación superior” en Comunicar, vol 51 , issue 2.

MARGALEF, L. \& ARENAS, A. (2006). “¿Qué entendemos por innovación educativa?. A propósito del desarrollo curricular” en Perspectiva Educacional, Formación de profesores, issue 47 p 13-31.

MORERA, \&. et al. (2013). Metodología innovadora y estrategias de aprendizaje en la Universidad. GIMA. Valencia: Universidad Politécnica de Valencia. http://doi.org/10.13140/2.1.1228.9609

QUIZHPE, L.A., GÓMEZ, O.A. \& AGUILAR, R.P. (2016). "La innovación educativa en la Educación Superior Ecuatoriana y el portafolio docente: instrumentos de desarrollo" en Revista Cubana de Reumatología, vol 18, issue 3, p 297-303.

RUÉ, J. (2007). Enseñar en la universidad. El EEES como reto para la educación superior. Madrid, ES: Narcea.

SALINAS, J. (2004). "Innovación docente y uso de las Tic en la enseñanza universitaria" en universities y Knowledge Society Journal, vol 1, issue 1.

SILVA, J. \& MATURANA, D. (2017). "Una propuesta de modelo para introducir las metodologías activas en la educación superior" en Innovación Educativa, vol 16, issues 73, p 117-131. 